\title{
A multiscale Mueller polarimetry module for a stereo zoom microscope
}

\author{
Adam Gribble $^{1} \cdot$ Michael A. Pinkert ${ }^{2,3,4} \cdot$ Jared Westreich ${ }^{1} \cdot$ Yuming Liu $^{2} \cdot$ Adib Keikhosravi $^{2,4}$. \\ Mohammadali Khorasani ${ }^{5}$. Sharon Nofech-Mozes ${ }^{6} \cdot$ Kevin W. Eliceiri $^{2,3,4} \cdot$ Alex Vitkin $^{1,7,8}$
}

Received: 19 February 2019 / Revised: 8 June 2019 / Accepted: 10 June 2019 / Published online: 20 June 2019

(c) Korean Society of Medical and Biological Engineering 2019

\begin{abstract}
Mueller polarimetry is a quantitative polarized light imaging modality that is capable of label-free visualization of tissue pathology, does not require extensive sample preparation, and is suitable for wide-field tissue analysis. It holds promise for selected applications in biomedicine, but polarimetry systems are often constrained by limited end-user accessibility and/ or long-imaging times. In order to address these needs, we designed a multiscale-polarimetry module that easily couples to a commercially available stereo zoom microscope. This paper describes the module design and provides initial polarimetry imaging results from a murine preclinical breast cancer model and human breast cancer samples. The resultant polarimetry module has variable resolution and field of view, is low-cost, and is simple to switch in or out of a commercial microscope. The module can reduce long imaging times by adopting the main imaging approach used in pathology: scanning at low resolution to identify regions of interest, then at high resolution to inspect the regions in detail. Preliminary results show how the system can aid in region of interest identification for pathology, but also highlight that more work is needed to understand how tissue structures of pathological interest appear in Mueller polarimetry images across varying spatial zoom scales.
\end{abstract}

Keywords Mueller matrix polarimetry $\cdot$ Multiscale $\cdot$ Stereo zoom microscope $\cdot$ Module $\cdot$ Pathology $\cdot$ Label-free imaging

\section{Introduction}

Polarimetry is a label-free optical imaging method with rich tissue biophysical information content that has potential for speeding up pathology assessments, but it is constrained by accessibility and long imaging times. Polarimetry uses polarized light to measure tissue structure and composition, which

Adam Gribble and Michael A. Pinkert have contributed equally to this work.

Kevin W. Eliceiri

eliceiri@wisc.edu

$\triangle$ Alex Vitkin

Alex.Vitkin@rmp.uhn.ca

1 Department of Medical Biophysics, University of Toronto, Toronto, Canada

2 Laboratory for Optical and Computational Instrumentation, Department of Biomedical Engineering, University of Wisconsin at Madison, Madison, USA

3 Department of Medical Physics, University of Wisconsin at Madison, Madison, USA change in many pathologies [1]. Studies using transmission geometries have shown that it can detect differences between benign and pathological tissue in breast [2], rectal [3], cervical [4], and liver cancers [5]. Polarimetry can perform these measurements on unstained tissue sections, bypassing a time-consuming step in the pathological pipeline. However, polarimetry systems are usually implemented on dedicated instruments, requiring specialized expertise and resources. In addition, complete polarized tissue characterization is performed via Mueller matrix (MM) polarimetry, which needs significantly

4 Morgridge Institute for Research, Madison, WI, USA

5 General Surgical Oncology Program, University of Toronto, Toronto, Canada

6 Department of Laboratory Medicine and Pathobiology, University of Toronto, Toronto, Canada

7 Division of Biophysics and Bioimaging, Princess Margaret Cancer Centre, University Health Network, Toronto, ON, Canada

8 Department of Radiation Oncology, University of Toronto, Toronto, Canada 
longer imaging + computation times since a minimum of 16 images using different input and output polarization states are required, followed by computational processing of the results [6]. This limitation highlights the need for new methods to speed up Mueller polarimetry imaging and processing times to make the polarimetric approach more practical for pathology adoption.

A polarimetry system that can perform spatial multiscale imaging (at multiple resolutions and fields-of-view, FOV) could offer significant and practical speed and tissue coverage benefits, and afford better alignment with current pathology practice. Pathologists perform slide analysis by first finding regions-of-interest (ROI) at low resolution overviews, and then zooming into examine them at high resolution [7]. Thus, only parts of a tissue sample need to be imaged at high resolution. Analogously, a multiscale polarimetry system could acquire low-resolution images of the whole tissue preparation, and then focus in on smaller FOV, high resolution images of chosen ROIs. This would significantly speed up the overall sample examination and image processing times. For example, a similar strategy successfully used polarimetry to speed up breast cancer diagnosis with mass spectrometry: finding ROIs with polarimetry and then analyzing these ROIs with the slower but more accurate mass spectrometry $[2,8,9]$. Thus, a multiscale polarimetry system would be a valuable tool for furthering basic research and potentially improving clinical utility of this promising polarized light approach.

This article addresses the issues of accessibility and long imaging time by describing a low-cost module for a stereo zoom microscope that converts it into a multiscale polarimetry system. The modularity allows the system to be added or removed from the stereo zoom microscope in less than a minute. The system's multiscale polarimetric zoom capability is demonstrated with images of human breast cancer samples, where a multiscale approach aids in delineating cancer ROIs. The article also describes the need for a better understanding of how tissue structures and pathologies appear across different spatial scales in polarimetry, using the example of murine breast cancer models. Here we focus on the structural protein fibrillar collagen, as it been shown to be a major pathology target in several cancers [10-19]. We compare multiscale MM polarimetric images to collagen-specific gold standard images obtained with second harmonic generation (SHG) microscopy [20]. Finally, the article discusses future directions for multiscale polarimetry.

\section{Methods}

\subsection{Multiscale Mueller polarimetry module}

Polarization properties of tissue are represented by the Mueller matrix, $\mathbf{M}$, which is a transfer function that describes how the polarization of light changes due to sample interaction. This is represented mathematically as

$\mathbf{S}_{\text {out }}=\mathbf{M S}_{\text {in }}$

where $\mathbf{S}_{\text {out }}$ is the output polarization state (output Stokes vector) and $\mathbf{S}_{\text {in }}$ is the input polarization state (input Stokes vector). Sample polarization properties, such as depolarization (a measure of tissue heterogeneity), linear retardance (related to tissue structural asymmetry), diattenuation (differential polarization absorption properties), and optical activity (influenced by the presence of chiral molecules, for example glucose) are contained within $\mathbf{M}$ and can be recovered using Mueller decomposition techniques. A popular $\mathrm{MM}$ analysis method is Lu-Chipman polar decomposition [21], which we use in the present paper; other decompositional approaches, and their relative advantages and disadvantages, are actively discussed in the literature but will not be covered here [22-28]. Briefly, the Mueller matrix $\mathbf{M}$, is decomposed into three matrices, describing depolarization $\mathbf{M}(\Delta)$, retardance $\mathbf{M}(\mathrm{R})$, and diattenuation $\mathbf{M}(\mathrm{D})$; from these three "basis" matrices, the sample biophysical polarization properties of interest are then calculated:

\section{$\mathbf{M}=\mathbf{M}(\Delta) \mathbf{M}(\mathrm{R}) \mathbf{M}(\mathrm{D})$.}

An experimental Mueller polarimetry system requires a polarization state generator (PSG) between the light source and sample to control the input polarizations, and a polarization state analyzer (PSA) between the sample and detector to measure the output polarizations. To successfully adapt a MM polarimetry module into a standard transmission microscope, the PSG must fit between light source and sample, and the PSA must fit within the microscope working distance (between sample and objective lens). These requirements present several challenges for imaging at multiple scales. First, changing magnifications usually requires switching lenses. Further, different lenses usually have different working distances. This means that a PSA designed for one objective lens may not be compatible with others. We addressed this by designing a polarimetry module for use with a commercial stereo zoom microscope (Axio Zoom. V16, Zeiss), as these types of microscopes perform continuous zooming without switching objective lenses. Further, the working distance does not change with magnifications, thus a single PSA can work across all magnifications. This enabled the development of a versatile multiscale MM polarimetry imager.

The polarimetry module is shown in Fig. 1. Both PSG and PSA were approximately $50 \mathrm{~mm}$ thick, suitable for incorporation into appropriate locations in the microscope's optical beam path. The PSG consists of a rotatable polarizer (LPVISE100-A, Thorlabs) followed by a rotatable quarterwave plate (QWP) (AQWP05M-600 or WPQ10M-633, Thorlabs). The PSA consists of an identical rotatable QWP 
followed by an identical rotatable polarizer. The polarization optics are placed in individual motorized angular rotation mounts (PRM1/MZ8, Thorlabs). Electromechanical controllers (KDC101, Thorlabs) for these mounts are connected to a computer, and rotation is enabled via a LabVIEW program. A stand holds the polarization optics over the translation stage, and a custom sample holder places the sample between the PSG and PSA. This configuration differs from typical MM microscopes, which position the
PSA between the microscope objective and the camera. Our placement of the PSA allows the module to be easily attached and removed from the microscope, an important practical consideration. One possible disadvantage of this configuration is the increase in measurement artefacts due to off-axis rays entering the PSA at a large angle. The microscope LED [Illuminator HXP 200C (D), Zeiss] is a $310 \mathrm{~W}$ uncollimated white light source passing through a filter centered at $630 \mathrm{~nm}$ (ET630/75 or ZET630/10, Chroma). The (a)

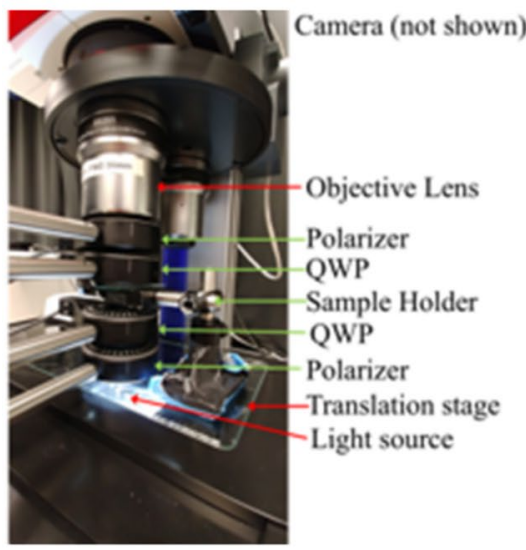

(b)

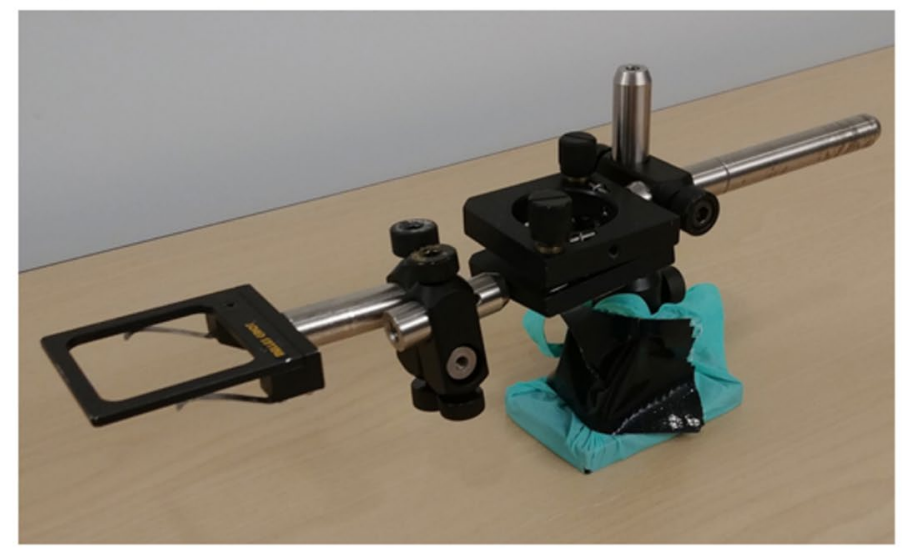

(c)

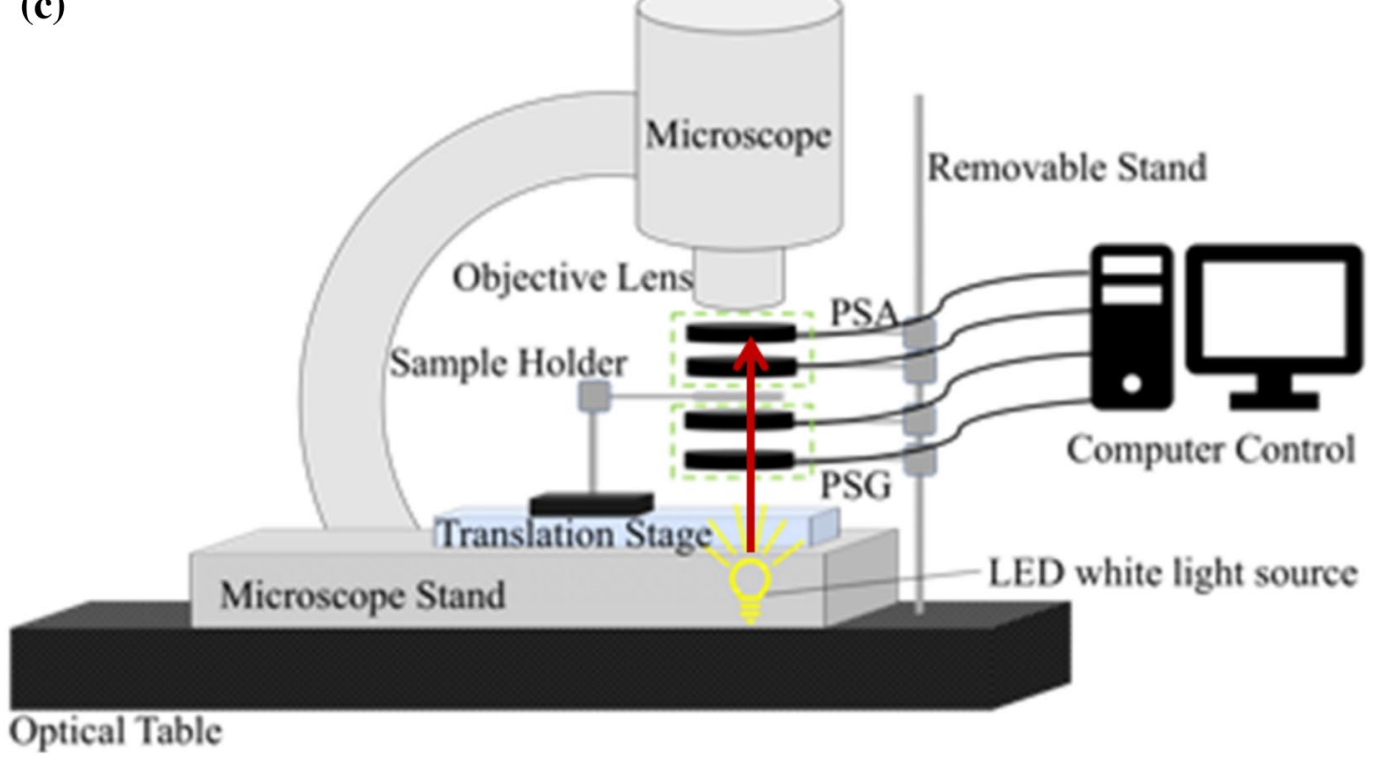

Fig. 1 Multiscale polarimetry module for use with a commercial stereo zoom microscope-a Photo of the polarimetry module. Light passes through a translation stage and polarization state generator (PSG), consisting of a linear polarizer and a quarter-wave plate (QWP). After interacting with the sample, light passes through a polarization state analyzer (PSA), consisting of QWP and linear polarizer. Finally, light is collected by the microscope objective lens and an image is recorded on a camera. Components of the polarimetry module are indicated by green arrows and components of the microscope are indicated by red arrows. b Custom sample holder coupled to the translation stage, with an arm that extends out between the PSG and PSA. This allows the sample to be placed in the focal plane and to be moved with the translation stage without interfering with the PSG and PSA. c Schematic of the set-up in (a). Each PSG and PSA component was attached to a horizontal post, which were all attached to a removable vertical stand. The stand was secured to the same optical table as the microscope. A sample holder sat on the microscope translation stage, extended over the imaging pathway, and could be moved with the translation stage if needed for image stitching at higher resolutions. The direction of the light path is indicated by the arrow. (Color figure online) 
filters are centered at $630 \mathrm{~nm}$ because tissue scattering and absorption due to hemoglobin are relatively low at longer visible wavelengths [29]. The broader $75 \mathrm{~nm}$ filter is used to increase signal for thicker samples. The $10 \mathrm{~nm}$ filter can increase the accuracy of linear retardance measurements and make it easier to relate linear retardance to birefringence for minimally scattering thin samples, as measurements of linear retardance are a weighted average across the wavelength range. It should be noted that birefringence varies by $\sim \pm 6 \%$ over the spectral band of the $75 \mathrm{~nm}$ filter and by $\sim \pm 0.8 \%$ over the spectral band of the $10 \mathrm{~nm}$ filter [30]. The camera (ORCA-Flash4.0 V3 Digital CMOS camera, Hamamatsu) has $2048 \times 2048$ pixels with a cell size of $6.5 \times 6.5 \mu \mathrm{m}$.

The system uses direct measurements of the Stokes vector to calculate the Mueller matrix. This requires 24 measured images instead of the minimum 16, but offers improved SNR [31]. Currently, the component rotation + image acquisition takes $10 \mathrm{~s}$ per image, yielding $4 \mathrm{~min}$ for all 24 images. The system was calibrated by measuring the input Stokes vectors at each pixel using a blank sample. The output Stokes vectors were then calculated with the samples in place. The calibration is done on a pixel-by-pixel basis in order to minimize spatially dependent distortions such as from the off-axis oblique rays. The Mueller matrix is then decomposed using Lu-Chipman decomposition to extract the linear retardance values $[6,32]$. There are many possible resultant polarimetric measures to choose from; linear retardance was chosen here for illustrative purposes because of its relative simplicity, and due to its direct biophysical interpretation in terms of sensitivity to aligned asymmetric (birefringent) tissue microstructures [6, 32].

The zoom microscope had an objective lens (PlanNeoFluar Z, Zeiss, NA=0.25) with a large working distance $(56 \mathrm{~mm})$. The fields-of-view ranged from $1.2 \times 1.2$ to $19 \times 19 \mathrm{~mm}^{2}$, with corresponding resolutions of $<2.2-31.2 \mu \mathrm{m}$. Specifications of the microscope at selected magnifications are listed in Table 1 . The resolutions of polarimetry images were tested using a resolution target (1951 USAF, R3L3S1N, Thorlabs) preceded by a QWP (AQWP05M-600, Thorlabs). The QWP had a manufacturer reported retardance of $\sim 97^{\circ}$. The system measured a retardance of $\sim 96^{\circ}$ (with a resolution of $5.5 \mu \mathrm{m}$ at $32 \times$ and $<2.2 \mu \mathrm{m}$ at $80 \times$ ). The reconstructed polarimetry images had resolutions worse than those of a single transmission image due to slight image shifts between polarization states. Rotating the PSG and PSA slightly alters the optical path due to minor angular variations in element thickness and/or optical properties, slightly shifting the image location and potentially moving it out of focus [33]. The shifts can be corrected by registering the 24 state images (Fig. 2), but the out-of-focus blurring limits the resolution. The shifts are constant at a particular magnification, so can be calculated once and used multiple times. We calculate the shifts, register the images, and save the results using a custom python package based on the Insight Toolkit [34]. It takes $\sim 25 \mathrm{~s}$ to calculate all 23 shifts. The image registration and saving takes $\sim 4 \mathrm{~s}$ for a $2048 \times 2048$ pixel image. The registered results are then entered into a Matlab script which reconstructs the Mueller matrix and performs Lu-Chipman decomposition on a $2048 \times 2048$ pixel MM image in $\sim 3.5$ min.

\subsection{Samples}

One of the advantages of MM polarimetry is that it can be done on unprocessed tissue samples with intrinsic polarimetric contrast, both in transmission and in reflection [9]. The samples in this study were thin sections prepared on slides to allow direct comparison between MM polarimetry and other modalities which do require preparation (SHG, $\mathrm{H} \& \mathrm{E}$ ), and to facilitate shipping between research sites. All reported MM polarimetry images were acquired on unstained samples. Data in Figs. 2, 4 and 5 were acquired using the AQWP05M-600 QWP and the ET630/75 filter. Data in Fig. 3 were acquired using the WPQ10M-633 QWP and the ZET630/10 filter.

1. After obtaining institutional ethics and biosafety approval, human breast cancer samples $(n=4)$ were obtained from Sunnybrook Hospital in Toronto, Ontario,
Table 1 Optical properties of multiscale polarimetry microscope at different magnifications

\begin{tabular}{lccccc}
\hline Magnification $^{\mathrm{a}}$ & NA & FOV $\left(\mathrm{mm}^{2}\right)$ & $\begin{array}{l}\text { Image resolu- } \\
\text { tion }(\mu \mathrm{m})^{\mathrm{b}}\end{array}$ & $\begin{array}{l}\text { Linear retardance reso- } \\
\text { lution in center }(\mu \mathrm{m})^{\mathrm{b}}\end{array}$ & $\begin{array}{l}\text { Linear retardance reso- } \\
\text { lution in corner }(\mu \mathrm{m})^{\mathrm{b}}\end{array}$ \\
\hline $7 \times$ & 0.03 & $18.9 \times 18.9$ & 13.9 & 31.2 & 35.1 \\
$16 \times$ & 0.07 & $8.2 \times 8.2$ & 6.2 & 7.8 & 8.8 \\
$32 \times$ & 0.13 & $4.1 \times 4.1$ & $<2.2$ & 5.5 & 5.5 \\
$80 \times$ & 0.24 & $1.65 \times 1.65$ & $<2.2$ & $<2.2$ & 3.1 \\
$112 \times$ & 0.25 & $1.18 \times 1.18$ & $<2.2$ & $<2.2$ & 2.5 \\
\hline
\end{tabular}

${ }^{a}$ Only five discrete magnifications are shown for illustration. However, the microscope is capable of continuous zoom over $7 \times-112 \times$ magnification

${ }^{\mathrm{b}} \mathrm{A}$ resolution of $<2.2 \mu \mathrm{m}$ indicates that all line pairs of the USAF resolution target could be resolved 
(a) Transmission overlay

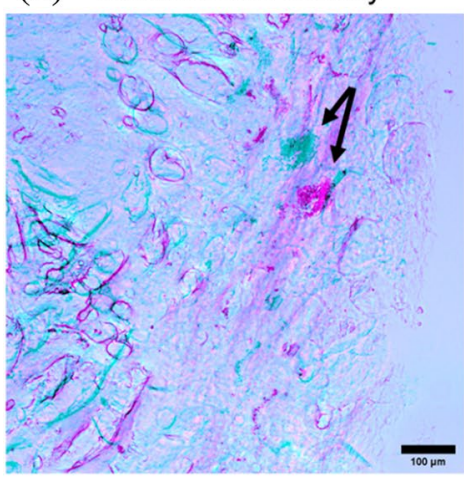

(b) Unregistered retardance

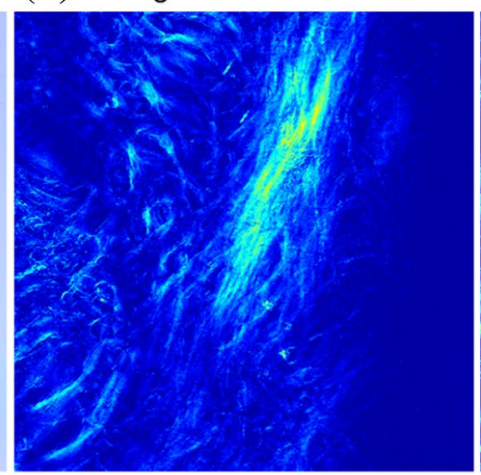

(c) Registered retardance

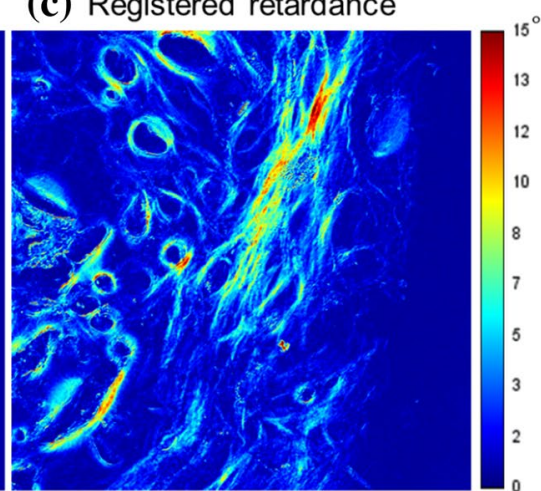

Fig. 2 Mueller matrix decomposition on a system with rotating optical elements requires registration between polarization statesImages of a PyVT mouse mammary gland from the same region of interest. a Overlay of two transmission images in cyan and magenta, taken at two of the 24 polarization states needed to reconstruct the MM, with black arrows indicating the same structure. b MM reconstruction of linear retardance without co-registering the 24 polarization images. c MM reconstruction of linear retardance after reg- istering the different the polarization state images. Rotating the polarization optical elements shifts the optical path and beam focus slightly [typical lateral displacements of the order of tens of microns, as in (a)], thus such MM systems need to register the images of each polarization state to achieve good image quality. The scale bar in (a) is $100 \mu \mathrm{m}$. The colorbar is in degrees linear retardance for images (b) and (c). (Color figure online)

Computational Instrumentation (LOCI), University of Wisconsin-Madison [37]. Briefly, this multiphoton system was built using an inverted microscope (Nikon, Diaphot 200) and a tunable Titanium:sapphire laser (Chameleon Ultra, Coherent). The laser was tuned to $890 \mathrm{~nm}$, an established standard for mammary gland SHG imaging [38]. The system uses a CFI Plan Apo 20X .75NA air immersion objective (Nikon). Backscattered SHG signal was collected with a H7422P-40 GaAsP photomultiplier detector (Hamamatsu) and filtered using a $445 \mathrm{~nm}$ band pass filter (445/20 BrightLine ${ }^{\circledR}$ singleband bandpass filter, Semrock). Image tiling was performed to cover the entire tissue slide. The image pixel size was $619 \mathrm{~nm}$. To cover the entire tissue thickness, images were acquired in z-stacks, with step sizes of $5 \mu \mathrm{m}$. Images were stitched together and maximum intensity projections were made from the entire z-stack (for direct comparison with polarimetric images). In addition, the laser intensity and photomultiplier gain were adjusted to maximize the dynamic range over the tiles without damaging the sample. Images were acquired using in-house developed WiscScan software.

\section{Results and discussion}

\subsection{Multiscale identification of ROls and pathological differentiation} rimetry, and four paired $\mathrm{H} \& \mathrm{E}$ were examined.

\subsection{Second harmonic generation microscopy}

SHG imaging was performed using a lab-built multiphoton microscope system at the Laboratory for Optical and
The main motivation for developing this MM polarimetric system was to speed up imaging tasks by identifying ROIs at low resolution and then imaging them at high resolution to provide better, more informative polarimetric views of 
(a) Transmission 16x

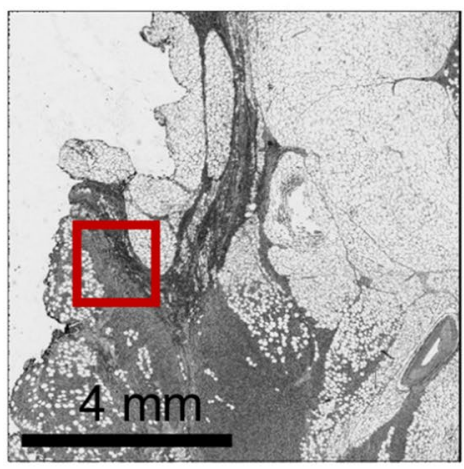

Transmission $112 x$

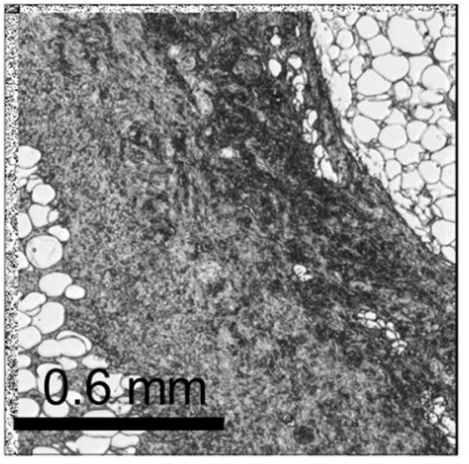

(b) Linear Retardance 16x

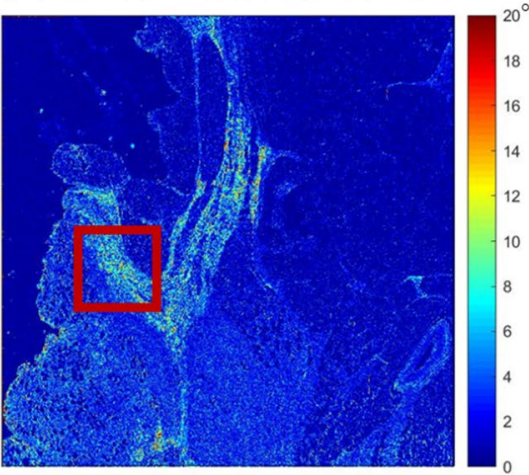

Linear Retardance $112 x$

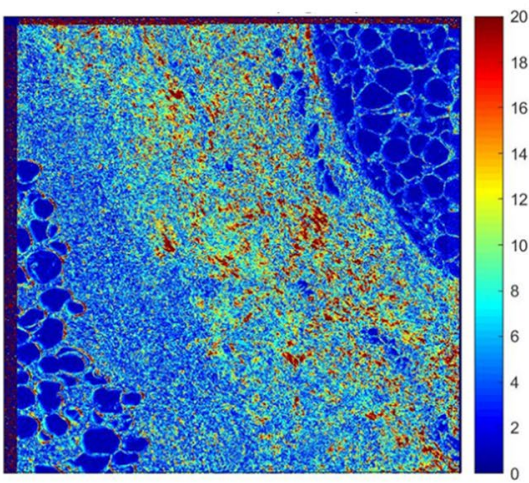

(c) H\&E 16x

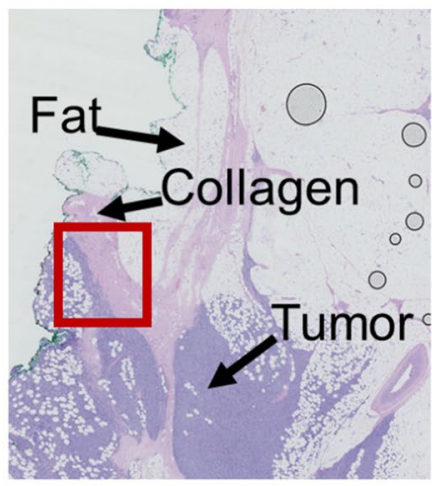

H\&E 112x

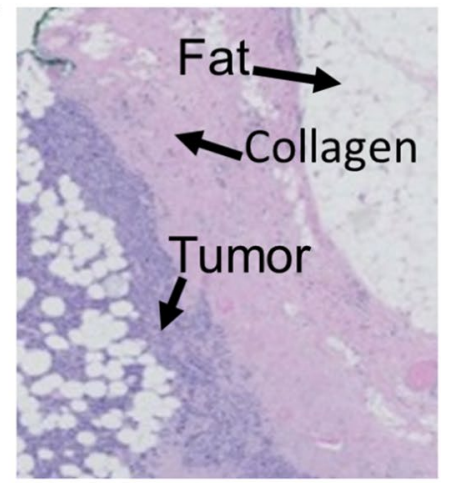

Fig. 3 Multiscale polarimetry of human infiltrative lobular carcinoma to identify and image ROIs. The top row is at a $\times 16$ magnification, with red boxes indicating the location of the $\times 112$ magnification images in the bottom row. a White light unpolarized transmission images. b Polarimetry images showing linear retardance. c H\&E images. Tumor tissue is not easily distinguished from collagen in unpolarized transmission images at either magnification. The lowresolution linear retardance and $\mathrm{H} \& \mathrm{E}$ images can identify regions of tumor and collagen, but lack details to definitively identify boundaries and region margins. In the high-resolution images, there are clear differences between regions of tumor, collagen, and fat. Note the good visual agreement between the unstained polarimetric linear retardance image and the stained $\mathrm{H} \& \mathrm{E}$ histology visualization. The scale bar for the top row is $4 \mathrm{~mm}$, and $0.6 \mathrm{~mm}$ for the bottom row. The color bars for (b) are of linear retardance in degrees. (Color figure online) the tissue microstructure. This zoom capability can greatly speed up imaging when only certain areas need to be looked at in detail, similar to a pathology scanning approach [7]. Analogous studies demonstrated this concept using polarimetric MM imaging to facilitate localized mass-spectrometry analysis of murine breast cancer samples [2, 8, 9]. Here, we tested this capability using human breast cancer samples, first imaged with polarimetry and then compared to pathologist's labeling. Representative images of a human infiltrative lobular breast carcinoma are displayed in Fig. 3. The sample was polarimetrically imaged at two magnifications ( $16 \times$ and $112 \times$, see Table 1 for specifications), with corresponding $\mathrm{H} \& \mathrm{E}$ histology. At the lower magnification, one can identify tumor regions using linear retardance that seems to agree with the H\&E image, but it is difficult to visualize tumor extent. At the higher magnification tumor, collagen, and healthy fat (lipid membranes) are visually distinguishable using linear retardance (the tissue asymmetry metric we selected here from many potential MM parameters, for illustrative purposes). These trends were observed in other human breast cancer samples as well. That is, lower magnification polarimetry can roughly identify regions of tumor (heterogeneity, increased depolarization) and collagen (asymmetry, increased linear retardance), but a higher magnification is needed to better distinguish their boundaries.

The MM polarimetry module also facilitates systematic studies on how tissue structures appear across multiple spatial scales. Such investigations are necessary owing to unique MM image formation principles that differ from other modalities. For example, one of the types of MM images (linear retardance shown in Fig. 3b) reports on tissue anisotropy within a pixel; this means that adjacent pixels at high resolution may have strong signals, but their anisotropy may average to a lower value at low resolution if the anisotropy directions or magnitudes vary significantly in adjacent 
pixels with respect to each other. This is distinct from fluorescence and similar optical techniques, where adjacent high signal pixels tend to average to a similarly high signal. This subtle difference in image formation as a function of resolution can have large effects when using MM polarimetry for interpreting, characterizing, and diagnosing tissue structure.

We sought to demonstrate the potential impact of multiscale polarimetry studies on tissue pathology through the example of collagen imaging. Collagen, as a key constituent of the extracellular matrix (ECM) and connective tissue, is one of the major landmarks pathologists use to navigate when examining tissue [39]. In addition, changes in fibrillar collagen organization are associated with many pathologies, including cancers of the lung [10], breast [11-14], pancreas $[15,16]$, ovary $[17,18]$, and brain [19]. These changes can be difficult to perceive by eye alone [14]. As such, there is interest in new methods, ideally label free, to image collagen organization and to monitor the changes in its amount and arrangement, potentially leading to discovery of new predictive and prognostic tools that can impact cancer management. MM polarimetry is one candidate; several groups have previously published on using it to detect collagen organization in tissues including human uterine [40, 41], human colon [42], and rat tail tendon [43]. However, MM polarimetry is not a collagen specific modality, and its reported linear retardance metric is sensitive to any aligned asymmetric tissue microstructure (e.g., cardiomyocyte fibers in the heart [44]). In addition, MM images may lose some of the aligned collagen signal due to the anisotropy averaging effect discussed above (if there are significant spatial gradients in its magnitude and/or direction, including (birefringence) variation in the important depth direction [45]). With our multiscale polarimetry system, we illustrate some of these effects in images of murine mammary gland samples using two resolutions of MM polarimetry and SHG (Fig. 4). SHG is a collagen-specific modality and currently serves as the gold standard for characterizing tissue fibrillar collagen. There are corresponding structures in all three images of Fig. 4, but different sizes of fibers are lost based on the resolution of the presented MM retardance image. In particular, regions of thin unaligned fibers are not visible on high-resolution MM retardance image $(80 \times$ magnification), and some thicker bundled fibers are lost at lower resolution $(32 \times$ magnification). Thus, in some ROIs and at some magnifications MM shows sensitivity to aligned collagen and may potentially serve for collagen analysis, but in other ROI selections at different magnifications the collagen fibers important to the imaging problem may be lost.

The effects of imaging resolution and collagen organization on image formation underscores the need for caution in interpreting some Mueller matrix imaging results; specific selections of resolutions and ROIs may affect the results and their interpretation. Indeed, the several studies quoted above that use polarimetry for collagen imaging [40-43] may need re-examination of the robustness of their findings in light of these FOV/resolution/resultant ROI selection considerations, and a multi-scale polarimetry module of the type described in this study may prove useful in such analysis. Thus, we posit that more research into what tissue structures appear in which tissue types and at what resolutions and in what MM images is warranted. This will allow more consistent and quantitative deployment of MM polarimetry in tissue characterization applications.

\subsection{Applications and future directions}

The multiscale MM polarimetry module represents an improvement in the capabilities of polarized light imaging for pathology assessment. The system allows one to get a rapid, large FOV overview of tissue pathology, before zooming into regions of interest to assess the detailed tissue polarization properties with finer resolution. In doing so, it facilitates studies on the polarimetric appearance of tissue structures across spatial scales. This approach in general, and the described polarimetry module in particular, can also be used to speed up imaging by rapidly identifying ROIs to probe with another slower but more accurate modalities (e.g., mass spectrometry). We've recently demonstrated the utility of this hybrid technology combination of MM imaging and mass spectrometry to accurate identification and assessment of breast cancer subtypes, speeding up slide examination times several-fold $[2,8,9]$. Analogously, once the confounding effects of resolution and ROI selection are properly analyzed and understood, polarimetry may potentially prove useful as an SHG guidance tool for more targeted collagen analysis.

This system will also be used for quantitative studies of MM polarimetry images. As other studies have identified [40, 42, 43], MM can quantitatively distinguish tissue characteristics in selected ROIs, but more work is needed to test the limits of sensitivity and precision across tissue types, pathologies, and tissue thicknesses [46]. Such studies will require whole tissue slide analysis to estimate results for all possible ROIs. This will possibly help transition to the clinic, as it will clarify the robustness of the results, which is an important measure when defining a quantitative tissue biomarker [47]. In addition, quantitative studies will examine more polarimetric parameters than discussed in this paper. The paper has shown images of linear retardance, but polarimetry also contains many other potential metrics of interest. (e.g., depolarization, fast-axis birefringence orientation, diattenuation, constituent Stokes vectors images, and others). For example, multiple studies have shown that depolarization can be used to differentiate between benign and pathologic tissue $[2,9,48]$. Slow-axis orientation correlates well to collagen fiber orientation when the fibers are thick 

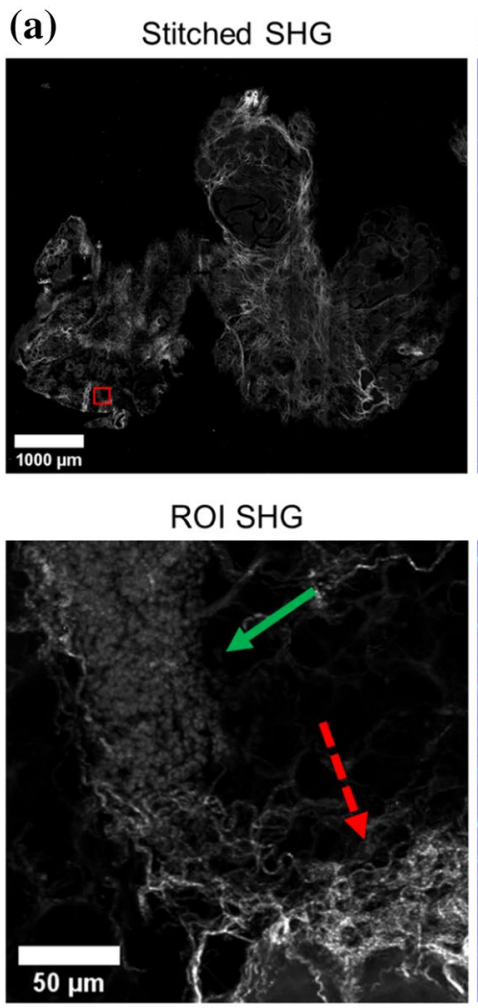

(b) Stitched 80x retardance

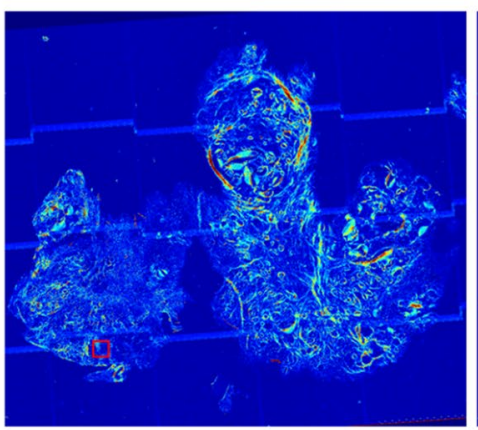

$\mathrm{ROI} 80 \mathrm{x}$ retardance

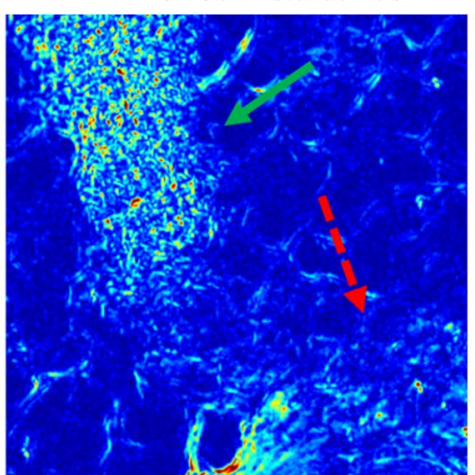

(c) Stitched $32 x$ retardance

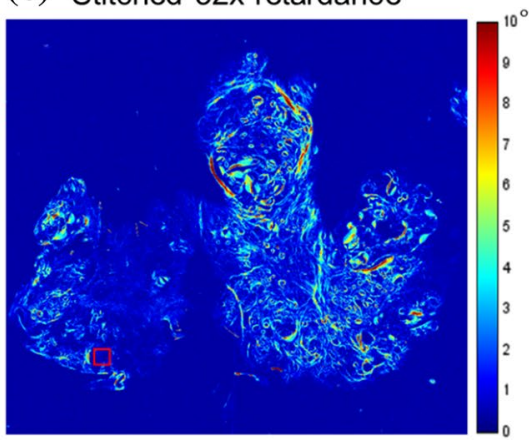

$\mathrm{ROI} 32 \mathrm{x}$ retardance

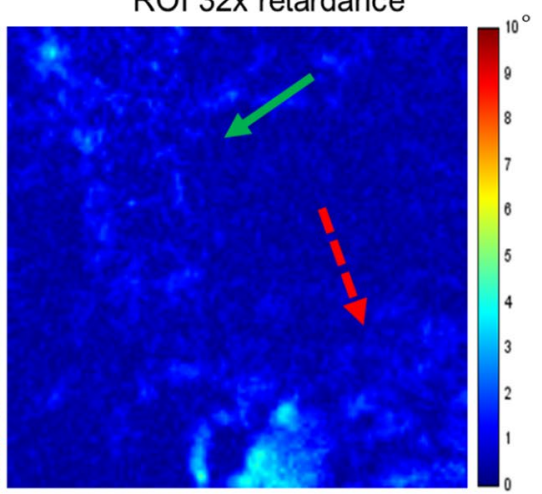

Fig. 4 The appearance of collagen in Mueller matrix polarimetry varies across spatial scales. The top row are multi-tile stitched images of a PyVT mouse mammary gland slide. All images were registered, and corresponding common ROI images (red squares in the top row) are shown in the bottom row. a Second harmonic generation microscopy images. b Polarimetry images at $\times 80$ magnification showing linear retardance. c Polarimetry images at $\times 32$ magnification showing linear retardance. The appearance of collagen fibers in MM polarimetry depends on their anisotropy within a resolution pixel: the

or bundled [40]. Diattenuation has shown less relevance to biological tissue, but may have potential in future investigations [32]. Further, the scientific and potentially clinical utility of multiscale polarimetry may be increased with the development of techniques for automatic tissue segmentation of various resultant MM images [48]. This can be done using traditional feature-based machine learning algorithms, or deep learning approaches which have recently become popular for medical image segmentation [49]. A potential advantage of polarimetry in the context of automatic segmentation is that there are many types of polarimetric images from which information can be obtained. For example, one can use machine learning techniques to combine the Mueller matrix images in ways that highlight different biological features [50]; future work will compare the ability of machine learning and traditional decomposition in this context (Fig. 5). More research is needed to determine which tissue types can be distinguished using polarimetry, which polarimetric images are optimum for this analysis, region of randomly oriented fibers depicted in (a) (pointed out by dashed red arrow) have little contrast in images (b) or (c); and an area of collagen fibers depicted in (a) (pointed out by solid green arrow) have strong contrast at the high resolution (b) but have weak contrast at the low resolution (c). Lines in top of (b) are artifacts from stitching together multiple tiles. The scale bar in (a) is $1000 \mu \mathrm{m}$ for the top row, $50 \mu \mathrm{m}$ for the bottom row. The color bars are of linear retardance in degrees for (b) and (c). (Color figure online)

and whether higher MM magnification results in increased segmentation accuracy. Finally, MM polarimetry is wavelength dependent and studies at additional wavelengths will be performed in the future. For example, multi-wavelength polarimetry is a promising approach to spectrally control depth of imaging/sampling volume in biological tissues [51].

The module itself can be improved in three aspects: the blurring of the resultant MM image compared to the unpolarized transmission image, acquisition time (10 s/ image $\times 24$ images $=4 \mathrm{~min}$ ), and the MM signal processing time ( $3.5 \mathrm{~min})$. The blurring of the MM image can be reduced through the use of Liquid Crystal (LC) variable retarders. The shifts in image location are caused by physically rotating QWPs and polarizers as described, which change the optical path slightly as a function of the rotation angle. LC retarders are solid-state alternatives and should not change the optical path. The LC retarders should also reduce the image acquisition time. For the current system, we are also investigating advanced co-registration methods 

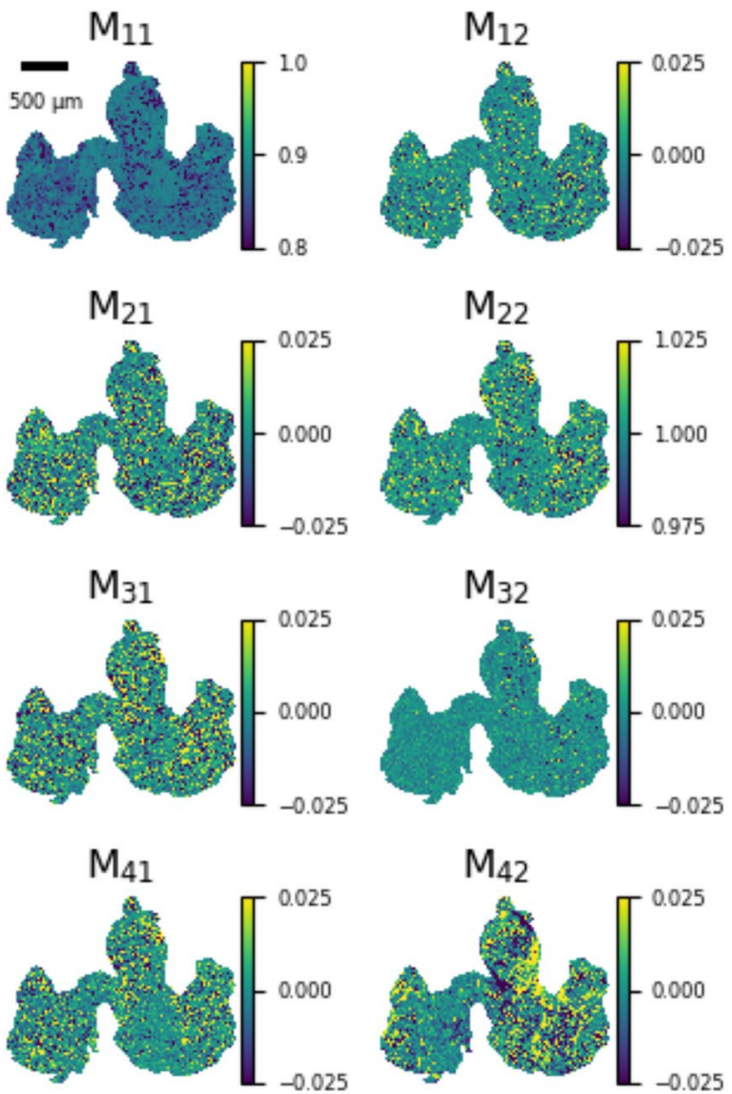

Fig. 5 Mueller matrix element images of sample in Fig. 4. The 16 elements of the Mueller matrix are difficult to interpret visually, thus many Mueller matrix imaging approaches rely on biophysical models to decompose the matrix into physical properties such as linear

as well as the effect of the number of measured images on resolution.

The LC retarders should also reduce the acquisition time. The current measurement protocol uses a fixed $10 \mathrm{~s}$ interval to allow all optical elements to settle and enable sufficient signal acquisition. The LC retarders change polarization states on the order of milliseconds, so would not need such a large $10 \mathrm{~s}$ interval. Further, while this calibration method measured Stokes vectors explicitly (requiring 24 total images), we are currently exploring an improved eigenvalue calibration method which only requires 16 images [52], based on an extension specifically suited for high NA optical systems [53]. Future work will investigate an optimal balance between speed/number of measured polarimetric images, resolution, and SNR.

Finally, the image processing time can also be addressed using different components. With current scripts, the calculation of a $2048 \times 2048$ pixel MM image requires approximately 3.5 min on an Intel i78086 K CPU. However, this time can be greatly reduced using Graphic Processing Units (GPU), as demonstrated in a recent study that achieved video-rate MM calculations [5].
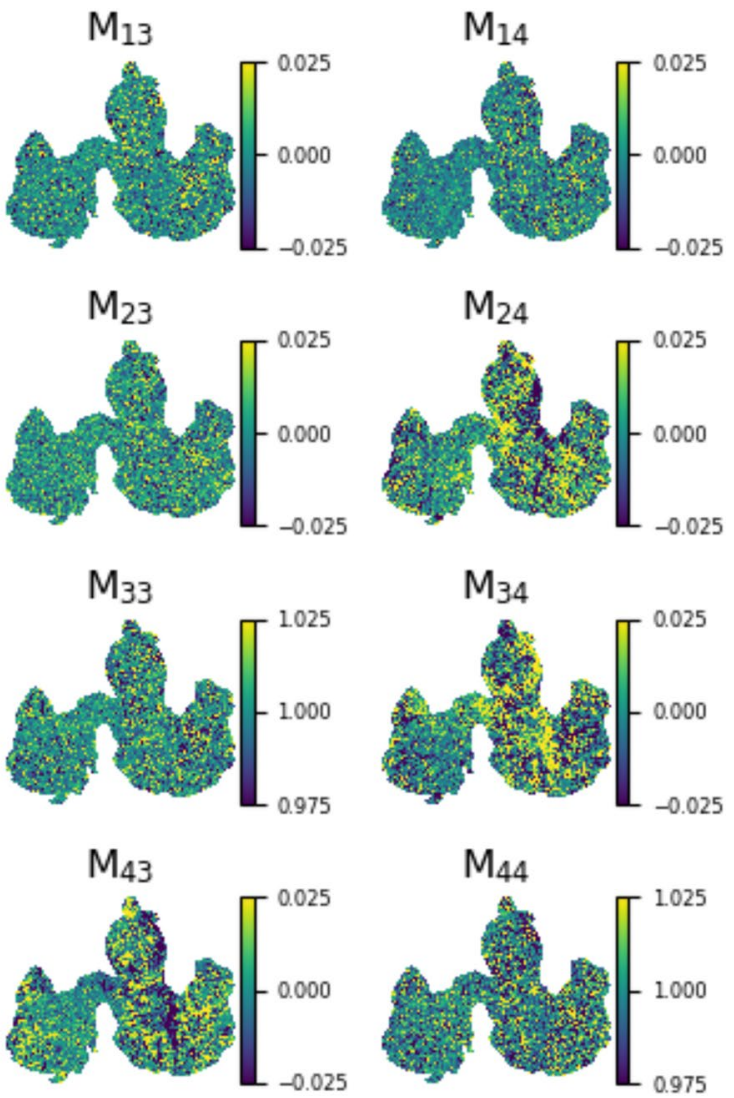

retardance, depolarization and diattenuation (for details, see text). Color bars have been adjusted for each element to better represent that element's numerical value, thus improving contrast. The scale bar in $M_{11}$ is $500 \mu \mathrm{m}$. (Color figure online)

\section{Conclusion}

This article described a multiscale Mueller matrix polarimetry module which can be easily and quickly coupled to a stereo zoom microscope. The module can smoothly change between imaging resolutions through a magnification range of $7 \times-112 \times$. This allows the use of low-resolution polarimetric images to find regions of interest for subsequent high-resolution detailed examination. This capability can significantly speed up imaging tasks and facilitates studies into the appearance of tissue microstructures across multiple imaging scales. Given the potential advantages of Mueller matrix polarimetric imaging-label-free methodology, modest sample preparation requirement, rich and relatively unexplored tissue biophysical information, wide field of view, rapid imaging times, robust signals, relatively simple and inexpensive instrumentation - a rigorous and robust examination of its strengths and limitations of the type reported in this article is warranted. Initial explorations in the preclinical models and human samples of breast cancer presented here demonstrate the bio-imaging potential of multiscale MM polarimetry and underscore some of its caveats. In 
particular, the variable-resolution MM polarimetric methodology should prove valuable for multiscale tissue studies using Mueller matrix imaging in pathology.

Acknowledgements The authors thanks David Inman and Dr. Suzanne Ponik of the UW-Madison for providing the murine mammary gland samples. Also, thanks are due to James Jonkman of the Advanced Optical Microscopy Facility (Toronto) for help with the stereo zoom microscope. We acknowledge Drs. Patricia Keely, Jeremy Bredfeldt, Joseph Szulczewski and Andreas Velten of the UW-Madison for useful discussions on the project. Funding is acknowledged from the Morgridge Institute for Research, Canadian Institutes of Health Research (AV) and NIH U54DK104310 (KWE). The authors also thank the UW Translational Research Initiatives in Pathology Laboratory, in part supported by the UW Department of Pathology and Laboratory Medicine and UWCCC Grant P30 CA014520, for use of its facilities and services.

Funding This study was funded by Morgridge Institute for Research (KWE), Canadian Institutes of Health Research (AV) and NIH U54DK104310 (KWE).

\section{Compliance with ethical standards}

Conflict of interest All authors declare that they have no conflicts of interest.

Ethical approval All applicable international, national, and/or institutional guidelines for the care and use of animals were followed. All procedures performed in studies involving human participants were in accordance with the ethical standards of the institutional and/or national research committee and with the 1964 Helsinki declaration and its later amendments or comparable ethical standards. Mouse mammary samples were obtained after acquiring approval from the Institutional Animal Care and Use Committee approval at the University of Wisconsin at Madison (WI, USA). The human breast samples were approved under institutional ethics and biosafety approval obtained from Sunnybrook Hospital in Toronto, Ontario, Canada.

Informed consent Sunnybrook REB determined that an informed consent form was not required for this study.

\section{References}

1. Qi J, Elson DS. Mueller polarimetric imaging for surgical and diagnostic applications: a review. J Biophotonics. 2017;10:950-82

2. Tata A, Gribble A, Ventura M, Ganguly M, Bluemke E, Ginsberg $\mathrm{HJ}$, et al. Wide-field tissue polarimetry allows efficient localized mass spectrometry imaging of biological tissues. Chem Sci. 2016;7:2162-9.

3. Ushenko YA, Sidor MI, Bodnar GB, Koval' GD. Mueller-matrix mapping of optically anisotropic fluorophores of biological tissues in the diagnosis of cancer. Quantum Electron. 2014;44:785.

4. Ushenko YA. Laser autofluorescence polarimetry of optically anisotropic structures of biological tissues in cancer diagnostics. Opt Spectrosc. 2015;118:1007-16.

5. Chang J, He H, Wang Y, Huang Y, Li X, He C, et al. Division of focal plane polarimeter-based $3 \times 4$ Mueller matrix microscope: a potential tool for quick diagnosis of human carcinoma tissues. J Biomed Opt. 2016;21:056002.
6. Ghosh N, Vitkin IA. Tissue polarimetry: concepts, challenges, applications, and outlook. J Biomed Opt. 2011;16:110801-11080129.

7. Krupinski EA, Tillack AA, Richter L, Henderson JT, Bhattacharyya $\mathrm{AK}$, Scott KM, et al. Eye-movement study and human performance using telepathology virtual slides. Implications for medical education and differences with experience. Hum Pathol. 2006;37:1543-56.

8. Tata A, Woolman M, Ventura M, Bernards N, Ganguly M, Gribble A, et al. Rapid detection of necrosis in breast cancer with desorption electrospray ionization mass spectrometry. Sci Rep. 2016;6:35374.

9. Woolman M, Gribble A, Bluemke E, Zou J, Ventura M, Bernards N, et al. Optimized mass spectrometry analysis workflow with polarimetric guidance for ex vivo and in situ sampling of biological tissues. Sci Rep. 2017;7:468.

10. Golaraei A, Cisek R, Krouglov S, Navab R, Niu C, Sakashita S, et al. Characterization of collagen in non-small cell lung carcinoma with second harmonic polarization microscopy. Biomed Opt Express. 2014;5:3562-7.

11. Golaraei A, Kontenis L, Cisek R, Tokarz D, Done SJ, Wilson $\mathrm{BC}$, et al. Changes of collagen ultrastructure in breast cancer tissue determined by second-harmonic generation double Stokes-Mueller polarimetric microscopy. Biomed Opt Express. 2016;7:4054-68.

12. Conklin MW, Eickhoff JC, Riching KM, Pehlke CA, Eliceiri $\mathrm{KW}$, Provenzano PP, et al. Aligned collagen is a prognostic signature for survival in human breast carcinoma. Am J Pathol. 2011;178:1221-32.

13. Li T, Sun L, Miller N, Nicklee T, Woo J, Hulse-Smith L, et al. The association of measured breast tissue characteristics with mammographic density and other risk factors for breast cancer. Cancer Epidemiol Biomark Prev. 2005;14:343-9.

14. Beck AH, Sangoi AR, Leung S, Marinelli RJ, Nielsen TO, van de Vijver MJ, et al. Systematic analysis of breast cancer morphology uncovers stromal features associated with survival. Sci Transl Med. 2011;3:108ra113.

15. Drifka CR, Loeffler AG, Esquibel CR, Weber SM, Eliceiri KW, Kao WJ. Human pancreatic stellate cells modulate 3D collagen alignment to promote the migration of pancreatic ductal adenocarcinoma cells. Biomed Microdevices. 2016;18:105.

16. Drifka CR, Loeffler AG, Mathewson K, Keikhosravi A, Eickhoff JC, Liu Y, et al. Highly aligned stromal collagen is a negative prognostic factor following pancreatic ductal adenocarcinoma resection. Oncotarget. 2016;7:76197-213.

17. Kirkpatrick ND, Brewer MA, Utzinger U. Endogenous optical biomarkers of ovarian cancer evaluated with multiphoton microscopy. Cancer Epidemiol Prev Biomark. 2007;16:2048-57.

18. Wen B, Campbell KR, Tilbury K, Nadiarnykh O, Brewer MA, Patankar M, et al. 3D texture analysis for classification of second harmonic generation images of human ovarian cancer. Sci Rep. 2016;6:35734.

19. Pointer KB, Clark PA, Schroeder AB, Salamat MS, Eliceiri KW, Kuo JS. Association of collagen architecture with glioblastoma patient survival. J Neurosurg. 2017;126:1812-21.

20. Campagnola P. Second harmonic generation imaging microscopy: applications to diseases diagnostics. Anal Chem. 2011;83:3224-31.

21. Lu S-Y, Chipman RA. Interpretation of Mueller matrices based on polar decomposition. J Opt Soc Am A. 1996;13:1106.

22. Ghosh N, Wood MFG, Li S, Weisel RD, Wilson BC, Li R-K, et al. Mueller matrix decomposition for polarized light assessment of biological tissues. J Biophotonics. 2009;2:145-56.

23. Ortega-Quijano N, Arce-Diego JL. Mueller matrix differential decomposition. Opt Lett. 2011;36:1942-4. 
24. Gil JJ, José IS, Ossikovski R. Serial-parallel decompositions of Mueller matrices. JOSA A. 2013;30:32-50.

25. Ossikovski R. Differential matrix formalism for depolarizing anisotropic media. Opt Lett. 2011;36:2330-2.

26. Martin L, Brun GL, Jeune BL, Pellen F, Rivet S. Analysis of experimental depolarizing Mueller matrices through a hybrid decomposition. Appl Opt. 2015;54:27-36.

27. Kumar S, Purwar H, Ossikovski R, Vitkin IA, Ghosh N. Comparative study of differential matrix and extended polar decomposition formalisms for polarimetric characterization of complex tissuelike turbid media. J Biomed Opt. 2012;17:105006.

28. Ghosh N, Wood MFG, Vitkin IA. Influence of the order of the constituent basis matrices on the Mueller matrix decompositionderived polarization parameters in complex turbid media such as biological tissues. Opt Commun. 2010;283:1200-8.

29. Jacques SL. Optical properties of biological tissues: a review. Phys Med Biol. 2013;58:R37.

30. Tuchin VV. Polarized light interaction with tissues. J Biomed Opt. 2016;21:071114.

31. Layden D, Wood MFG, Vitkin IA. Optimum selection of input polarization states in determining the sample Mueller matrix: a dual photoelastic polarimeter approach. Opt Express. 2012;20:20466-81.

32. Alali S, Vitkin A. Polarized light imaging in biomedicine: emerging Mueller matrix methodologies for bulk tissue assessment. J Biomed Opt. 2015;20:061104.

33. Smith MH, Woodruff JB, Howe JD. Beam wander considerations in imaging polarimetry. In: SPIE Polarization: measurement, analysis, and remote Sensing II. 1999; p. 50-5.

34. Yoo TS, Ackerman MJ, Lorensen WE, Schroeder W, Chalana V, Aylward S, et al. Engineering and algorithm design for an image processing API: a technical report on ITK - the Insight Toolkit. Stud Health Technol Inf. 2002;85:586-92.

35. Fantozzi A, Christofori G. Mouse models of breast cancer metastasis. Breast Cancer Res. 2006;8:212.

36. Lin EY, Jones JG, Li P, Zhu L, Whitney KD, Muller WJ, et al. Progression to malignancy in the polyoma middle $\mathrm{T}$ oncoprotein mouse breast cancer model provides a reliable model for human diseases. Am J Pathol. 2003;163:2113-26.

37. Bird DK, Eliceiri KW, Fan C-H, White JG. Simultaneous twophoton spectral and lifetime fluorescence microscopy. Appl Opt. 2004;43:5173-82.

38. Provenzano PP, Eliceiri KW, Campbell JM, Inman DR, White JG, Keely PJ. Collagen reorganization at the tumor-stromal interface facilitates local invasion. BMC Med. 2006;4:38.

39. Carwile Leroy E. Collagen in the physiology and pathology of connective tissue. Steffan Gay and Edward J. Miller. New York, Gustav Fischer Inc., 1978. 106 pages; illustrated. price: \$21.10. Arthritis Rheum. 1980;23:134-5.

40. Bancelin S, Nazac A, Ibrahim BH, Dokládal P, Decencière E, Teig B, et al. Determination of collagen fiber orientation in histological slides using Mueller microscopy and validation by second harmonic generation imaging. Opt Express. 2014;22:22561-74.
41. Nazac A, Bancelin S, Teig B, Ibrahim BH, Fernandez H, SchanneKlein M-C, et al. Optimization of Picrosirius red staining protocol to determine collagen fiber orientations in vaginal and uterine cervical tissues by Mueller polarized microscopy. Microsc Res Tech. 2015;78:723-30.

42. Keikhosravi A, Liu Y, Drifka C, Woo KM, Verma A, Oldenbourg $\mathrm{R}$, et al. Quantification of collagen organization in histopathology samples using liquid crystal based polarization microscopy. Biomed Opt Express. 2017;8:4243-56.

43. Ellingsen PG, Aas LMS, Hagen VS, Kumar R, Lilledahl MB, Kildemo M. Mueller matrix three-dimensional directional imaging of collagen fibers. J Biomed Opt. 2014;19:026002.

44. Wood MFG, Ghosh N, Wallenburg MA, Li S-H, Weisel RD, Wilson BC, et al. Polarization birefringence measurements for characterizing the myocardium, including healthy, infarcted, and stem-cell-regenerated tissues. J Biomed Opt. 2010;15:047009.

45. Alali S, Wang Y, Vitkin IA. Detecting axial heterogeneity of birefringence in layered turbid media using polarized light imaging. Biomed Opt Express. 2012;3:3250-63.

46. Dong Y, Qi J, He H, He C, Liu S, Wu J, et al. Quantitatively characterizing the microstructural features of breast ductal carcinoma tissues in different progression stages by Mueller matrix microscope. Biomed Opt Express. 2017;8:3643-55.

47. Sullivan DC, Obuchowski NA, Kessler LG, Raunig DL, Gatsonis C, Huang EP, et al. Metrology standards for quantitative imaging biomarkers. Radiology. 2015;277:813-25.

48. Ahmad I, Gribble A, Murtza I, Ikram M, Pop M, Vitkin A. Polarization image segmentation of radiofrequency ablated porcine myocardial tissue. PLoS ONE. 2017;12:e0175173.

49. Komura D, Ishikawa S. Machine learning methods for histopathological image analysis. Comput Struct Biotechnol J. 2018;16:34-42.

50. Li X, Liao R, Zhou J, Leung PTY, Yan M, Ma H. Classification of morphologically similar algae and cyanobacteria using Mueller matrix imaging and convolutional neural networks. Appl Opt. 2017;56:6520-30.

51. Pierangelo A, Manhas S, Benali A, Fallet C, Antonelli M-R, Novikova T, et al. Ex vivo photometric and polarimetric multilayer characterization of human healthy colon by multispectral Mueller imaging. J Biomed Opt. 2012;17:066009.

52. Compain E, Poirier S, Drevillon B. General and self-consistent method for the calibration of polarization modulators, polarimeters, and Mueller-matrix ellipsometers. Appl Opt. 1999;38:3490-502.

53. Macias-Romero C, Török P. Eigenvalue calibration methods for polarimetry. J Eur Opt Soc-Rapid Publ. 2012;7:12004.

Publisher's Note Springer Nature remains neutral with regard to jurisdictional claims in published maps and institutional affiliations. 